\title{
New method for determining free core nutation parameters, considering geophysical effects
}

\begin{abstract}
J. Vondrák and C. Ron
Astronomical Institute CAS, Boční II, 14100 Prague 4, Czech Republic

e-mail:vondrak@ig.cas.cz, ron@asu.cas.cz

Received 16 February 2017 / Accepted 26 June 2017

ABSTRACT

Context. In addition to the torques exerted by the Moon, Sun, and planets, changes of precession-nutation are known to be caused also by geophysical excitations. Recently studies suggest that geomagnetic jerks (GMJ) might be associated with sudden changes of phase and amplitude of free core nutation. We showed that using atmospheric and oceanic excitations with those by GMJ improves substantially the agreement with observed celestial pole offsets.

Aims. Traditionally, the period $T_{f}$ and quality factor $Q_{f}$ of the free core nutation (FCN) are derived from VLBI-based celestial pole offsets (CPO). Either direct analysis of the observed CPO, or indirect method using resonant effects of nutation terms with frequencies close to FCN, are used. The latter method is usually preferred, since it yields more accurate results. Our aim is to combine both approaches to better derive FCN parameters.

Methods. We numerically integrated the part of CPO that is due to geophysical excitations for different combinations of $T_{f}, Q_{f}$, using Brzeziński's broadband Liouville equations (Brzeziński 1994, Manuscripta geodaetica, 19, 157), and compared the results with the observed values of CPO. The values yielding the best fit were then estimated. The observed CPO, however, must be corrected for the change of nutation that is caused by the $T_{f}, Q_{f}$ values different from those used to calculate IAU 2000 model of nutation. To this end, we have used the Mathews-Herring-Buffet transfer function and applied it to the five most affected terms of nutation (with periods $365.26,182.62,121.75,27.55$ and 13.66 days).

Results. The results, based on the CPO data in the interval 1986.0 - 2016.0 and excitations with three different models, are presented. We demonstrate that better results are obtained if the influence of additional excitations at GMJ epochs is added to excitations by the atmosphere and oceans. Our preferred values are $T_{f}=430.28 \pm 0.04$ mean solar days and $Q_{f}=19500 \pm 200$.
\end{abstract}

Key words. astrometry - reference systems - Earth - techniques: miscellaneous - methods: numerical

\section{Introduction}

The motion of the Earth axis in the celestial reference frame is mostly long-periodic motion, called precession-nutation, which is driven by external gravitational torque and can be well predicted. The free term, free core nutation $(\mathrm{FCN})$ is the rotational mode of the Earth's outer core caused by the misalignement of the rotation axis of the mantle and outer core. In celestial reference frame it is a retrograde long-periodic motion (appearing in terrestrial frame as nearly diurnal free wobble, hereafter NDFW). Nutation is thus a very important part of the transformation between terrestrial and celestial reference systems. Exact knowledge of FCN parameters is crucial in computing the reaction of the rotating Earth to external torques exhibited by other solar system bodies, namely the Moon, Sun and planets. The modification of both amplitudes and phases of individual nutation terms, depending on FCN parameters and their frequencies, are discussed below in Sect. 2, namely by Eq. (5). It is also necessary to say that the $\mathrm{FCN}$ period $T_{f}$ is closely related to the ellipticity of the core and electromagnetic coupling on the core-mantle boundary. $Q_{f}$ describes the speed of damping of the FCN amplitude. Without any excitation, the amplitude would exponentially decrease to zero; the larger is $Q_{f}$, the slower is the damping.

Especially now, when ESA mission Gaia is going to provide unprecedentedly accurate celestial reference frame, further improvement of precession-nutation model is becoming a necessity. To this end, IAU Commission A2 Working Group Theory of Earth Rotation and Validation has been set up. This study is our contribution to these efforts. Since the same laws apply to the rotation of all solid celestial bodies, our results might be interesting also as a reference for exoplanets studies.

In history, different observation techniques and methods of determining FCN parameters (period, quality factor) were used. Here follows an overview of some of the results obtained so far.

Before the new precise nutation model was derived, FCN parameters were determined by geophysical methods from gravimetric observations by superconducting gravimeters (Defraigne et al. 1999). The resonance due to the NDFW, observed in the diurnal tesseral tides and in the associated nutations, is used. The analysis of tidal data series yields the values of period $T_{f}=431 \pm 0.9$ sidereal days and quality factor $Q=54000(36000 ; 109000)$.

Florsch \& Hinderer (2000) derived FCN parameters from tidal gravity data in 1988-1996 using a Bayesian inversion method. The period obtained is 428 days, and $Q_{f}$ around $10^{5}$. They pointed out the important impact of inaccurate ocean loading corrections on the determination of the damping of the eigenmode; wrong ocean corrections may lead to underestimation of $Q_{f}$.

Hinderer et al. (2000) analyzed VLBI and gravimetric data, and showed that the apparent variations appearing in the 
eigenperiod and quality factor of $\mathrm{FCN}$ are related to a variable noise level in the tidal gravimetric data. From analysis of VLBI data they concluded that the amplitude variations found in the forced nutational response of the Earth are not real but rather induced by a variable free mode excitation. They showed that the Eigenperiod is stable within a range of three days.

Recently very accurate IAU models of nutation IAU 2000 (Mathews et al. 2002) and precession IAU 2006 (Capitaine \& Wallace 2006) appeared and were adopted as standard by the IAU. VLBI observations of celestial pole offsets (CPO) enable to determine, among others, also FCN parameters. Four free modes were used in their Mathews-Herring-Buffet (MHB) transfer function, among which $T_{f}=430.21 \pm 0.28$ days and $Q_{f}=20000$ were used.

Vondrák et al. (2005) compared direct and indirect method of determining FCN period from a combination of VLBI with GPS observations of CPO in 1984-2004. They found that while the direct method yields large variations of the period, 435460 days, the indirect method through resonances gives a very stable, more precise and much smaller value $T_{f}=430.55 \pm$ 0.11 days. The difference is ascribed to geophysical excitations that might influence direct determination.

Rosat \& Lambert (2009) studied sensitivity of VLBI and gravimetric observations to FCN parameters. They analyzed data from 15 superconducting gravimeters (SG) and VLBI delays accumulated over the preceding 24 yr. The estimated period and quality factor are consistent for both techniques. The inversion leads to a mean value of quality factor $\approx 16600( \pm 3500$ from SG and \pm 900 from VLBI), and to a resonant period in the range 423.3-430.5 days for SG and 427.8-431.4 days for VLBI within the $3 \sigma$ interval.

Gubanov (2010) analyzed VLBI observations over 25 yr. An amplitude-phase analysis of this series allowed to show that the FCN period changed several times. It was 418.1 days before 1992.1 and then 431.6 days until 1999.0. In 1999, the oscillations damped out almost completely and the process was restructured. Since 2000, the amplitude of these oscillations increased and their period became 450.7 days.

Huang et al. (2011) tested the FCN period with and without electromagnetic coupling (EMC). They received the period without EMC 433.52, with EMC 433.14 sidereal days. Koot \& de Viron (2011) obtained, from inversion of nutation observations and using atmosphere from NCEP reanalysis, the values of period and $Q_{f}$ without atmosphere $(429.09,19641)$, with a mean atmosphere $(429.55,19416)$ and with the variable atmosphere $(429.82,19042)$, respectively.

Amoruso et al. (2012) derived FCN parameters from strain data from the eight-year observations at Gran Sasso extensometers. The analyses led to the period of the FCN about 429 sidereal days, and the quality factor was not well constrained; however their results are consistent with recently published values $(\approx 20000)$.

Krásná et al. (2013) estimated FCN period within a global VLBI solution (1984-2011) from solid Earth tidal displacement as $431.23 \pm 2.44$ sidereal days and from the $\mathrm{CPO}$ as $431.17 \pm 0.09$ sidereal days. The final value for the FCN period was derived from the solid Earth tidal displacement and from the CPO in a joint solution. Its estimated value of $431.18 \pm 0.10$ sidereal days differs slightly from the conventional value 431.39 sidereal days given in IERS Conventions 2010 (Petit \& Luzum 2010).

Chao \& Hsieh (2015) estimated the eigenperiod of FCN from the VLBI data using the reverse, deconvolution process. They obtained the eigenperiod $T_{f}=441 \pm 4.5$ sidereal days, where $1 \sigma$ uncertainty is assessed via extensive Monte Carlo simulations.

Zhou et al. (2016) estimated the FCN period by the slidingwindow complex least-squares fit method, as an alternative direct method. The estimated values of the FCN period for the full set of 1984.0-2014.0 and four subsets range from 428.8 to 434.3 mean solar days.

Rapid changes of amplitude and phase of the retrograde free term, FCN, occur near the epochs of geomagnetic jerks (GMJ), as recently demonstrated, for example, by Malkin (2013). Vondrák \& Ron (2015) showed that better agreement with observations is obtained if additional impulse-like excitations due to GMJ are added to the effects of geophysical fluids.

Here we propose a new method of determining period and quality factor of FCN $\left(T_{f}, Q_{f}\right)$, using the numerical integration of broadband Liouville equations (Brzeziński 1994) with the geophysical excitations. Parameters $T_{f}, Q_{f}$ are important when constructing a nutation theory - they have a significant role in MHB transfer function (see Mathews et al. 2002). It expresses a ratio between the non-rigid and rigid Earth solution; in the presently adopted nutation model IAU 2000, rigid Earth solution by Souchay et al. (1999) is used.

\section{New method of determining $T_{f}, Q_{f}$}

Usually, the FCN period and quality factor are determined from observed celestial pole offsets by one of the following methods (see, e.g., Vondrák et al. 2005):

- directly, either by spectral analysis of the observed celestial pole offsets (CPO) or by fitting a least-squares sinusoid to CPO. These methods are usually less precise than

- indirect methods, using resonance effects in observed nutation terms close to retrograde FCN frequency. Corresponding nutation terms are determined from the observed CPO and divided by the rigid-Earth values. Then, the values of $T_{f}, Q_{f}$ are found that lead to a MHB transfer function (see Eq. (5) and Fig. 1 below) yielding a best fit to these ratios.

VLBI-based CPO (differences between observed and modeled precession-nutation) are a mixture of deficiency of the adopted IAU nutation model, geophysical excitations, and a free term (FCN). The latter is not a part of the model, because it is in principle non-predictable and as such it must be determined from the observations. The adopted model of nutation IAU 2000 contains, apart from external torques, also a simplified model of geophysical excitation (so called "Sun-synchronous correction") with prograde annual frequency (see Mathews et al. 2002, Table 7). This empirical term was added to the model to get rid of systematic residuals that the authors found when comparing their theoretical model with observations.

We have followed a procedure similar to the one that we used to derive parameters of Chandler wobble (Vondrák et al. 2017), but the situation here is more complicated because of the strong resonances that must be considered. Thus we propose a combination of direct and indirect approach. We are looking for the best fit (in least-squares sense) to the observed CPO, and at the same time we account for resonance effects that modify nutation and, consequently, also CPO. The main ideas of the new method are as follows:

- We removed Sun-synchronous correction from CPO, and compare the result with the result of numerical integration of broadband Liouville equations (Brzeziński 1994), using 


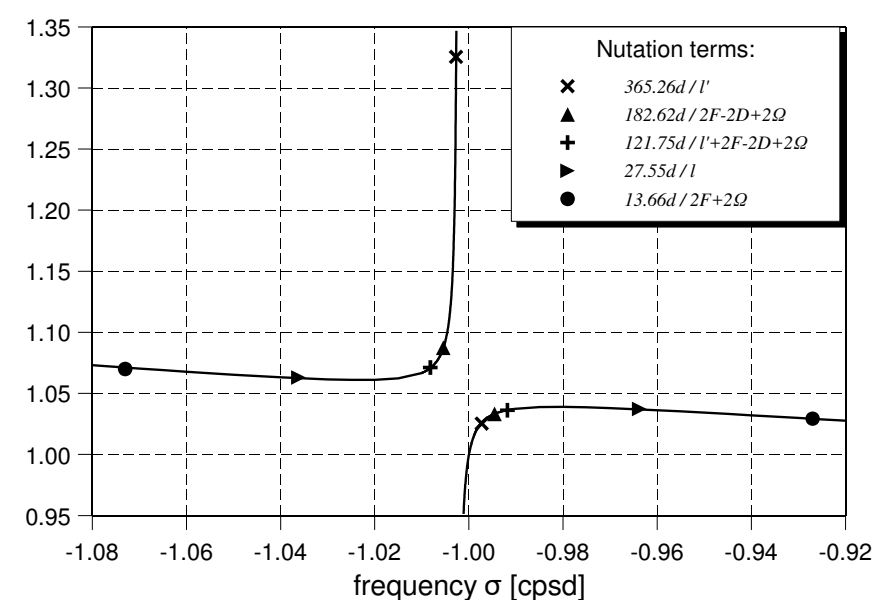

Fig. 1. Real part of MHB transfer function and five most influenced nutation terms.

atmospheric and oceanic excitations. Alternatively, we also added impulse-like excitations at the epochs of GMJ.

- We have done this for many combinations of $T_{f}, Q_{f}$ and, since nutation depends on these parameters, we corrected CPO for the difference between these values $T_{f}, Q_{f}$ and those used to construct the IAU model $\left(T_{f}=430.21 \mathrm{~d}\right.$, $\left.Q_{f}=20000\right)$. To this end, we applied MHB transfer function (Mathews et al. 2002) for the five most sensitive nutation terms (with periods 365.26, 182.62, 121.75, 27.55 and 13.66 days).

- We then looked for the combination $T_{f}, Q_{f}$ yielding the best rms fit between integration and observed CPO.

To achieve this, we used the following formulae:

1. Broadband Liouville equations in complex form, describing the relation between geophysical excitations and celestial pole offsets

$$
\begin{aligned}
\ddot{P} & -\mathrm{i}\left(\sigma_{\mathrm{C}}^{\prime}+\sigma_{f}^{\prime}\right) \dot{P}-\sigma_{\mathrm{C}}^{\prime} \sigma_{f}^{\prime} P= \\
& -\sigma_{\mathrm{C}}\left\{\sigma_{f}^{\prime}\left(\chi_{p}^{\prime}+\chi_{w}^{\prime}\right)+\sigma_{\mathrm{C}}^{\prime}\left(a_{p} \chi_{p}^{\prime}+a_{w} \chi_{w}^{\prime}\right)\right. \\
& \left.+\mathrm{i}\left[\left(1+a_{p}\right) \dot{\chi}_{p}^{\prime}+\left(1+a_{w}\right) \dot{\chi}_{w}^{\prime}\right]\right\},
\end{aligned}
$$

in which $P=\mathrm{d} X+\mathrm{id} Y \approx \Delta \psi \sin \epsilon_{0}+\mathrm{i} \Delta \epsilon$ denotes the part of $\mathrm{CPO}$, caused by geophysical excitation $(\Delta \psi, \Delta \epsilon$ are nutation offsets in longitude and obliquity, respectively), $\sigma_{\mathrm{C}}$ is the prograde Chandler frequency in terrestrial frame. $\sigma_{\mathrm{C}}^{\prime}, \sigma_{f}^{\prime}$ are the Chandler and retrograde FCN frequencies, and $\chi_{p}^{\prime}, \chi_{w}^{\prime}$ are the effective angular momentum functions (pressure and wind terms, respectively), all in celestial frame. All frequencies $\sigma$ are expressed in radians per day. The relation between FCN frequency $\sigma_{f}^{\prime}$ and its period $T_{f}$ (expressed in mean solar days) and quality factor $Q_{f}$ is given by the expression

$\sigma_{f}^{\prime}=-\frac{2 \pi}{T_{f}}\left[1-\mathrm{i}\left(1+1.00273 T_{f}\right) / 2 Q_{f}\right]$.

Numerical constants $a_{p}=9.200 \times 10^{-2}, a_{w}=2.628 \times 10^{-4}$, expressing different reaction on pressure and wind terms, are taken from Koot \& de Viron (2011). There is a simple relation between a frequency in terrestrial and celestial reference frames, $\sigma^{\prime}=\sigma+\Omega$, where $\Omega=6.30038 \mathrm{rad} /$ day is the mean speed of rotation of the Earth. Here we use complex Chandler frequency $\sigma_{\mathrm{C}}^{\prime}=6.314935+0.000208 \mathrm{i}$, based on Chandler wobble parameters derived recently by Vondrák et al. (2017). The complex effective angular momentum functions $\chi=\chi_{1}+\mathrm{i} \chi_{2}$ in terrestrial frame (their definition being given by Barnes et al. 1983) can be transformed into celestial frame, using a simple formula $\chi^{\prime}=-\chi \mathrm{e}^{\mathrm{i} \phi}$, where $\phi$ is the Greenwich sidereal time; retrograde quasidiurnal signal in terrestrial frame becomes long-periodic in celestial frame.

The homogeneous solution to Eq. (1), in which the righthand-side is put equal to zero, is $P=a_{\mathrm{C}} \exp \left(\mathrm{i} \sigma_{\mathrm{C}}^{\prime} t\right)+$ $a_{f} \exp \left(\mathrm{i} \sigma_{f}^{\prime} t\right)$, describing two free motions with complex amplitudes $a_{\mathrm{C}}, a_{f}$ and frequencies $\sigma_{\mathrm{C}}^{\prime}, \sigma_{f}^{\prime}$, respectively. The amplitudes are tied to the initial pole position $P(0)$ and its first time derivative $\dot{P}(0)$ by expressions

$a_{\mathrm{C}}=\left(\sigma_{f}^{\prime} P(0)+\mathrm{i} \dot{P}(0)\right) /\left(\sigma_{f}^{\prime}-\sigma_{\mathrm{C}}^{\prime}\right)$

$a_{f}=\left(\sigma_{\mathrm{C}}^{\prime} P(0)+\mathrm{i} \dot{P}(0)\right) /\left(\sigma_{\mathrm{C}}^{\prime}-\sigma_{f}^{\prime}\right)$.

To numerically integrate Eq. (1), a two-order differential equation in complex form, we must split it into a system of two first-order equations. We have done so by introducing substitutions, containing new variables $Y_{1}=P$ and $Y_{2}=\dot{P}-\mathrm{i} \sigma_{\mathrm{C}}^{\prime} P$. They lead to equations, easily integrable by standard procedures:

$$
\begin{aligned}
\dot{Y}_{1}= & \mathrm{i} \sigma_{\mathrm{C}}^{\prime} Y_{1}+Y_{2} \\
\dot{Y}_{2}= & \mathrm{i} \sigma_{f}^{\prime} Y_{2}-\sigma_{\mathrm{C}}\left\{\sigma_{f}^{\prime}\left(\chi_{p}^{\prime}+\chi_{w}^{\prime}\right)+\sigma_{\mathrm{C}}^{\prime}\left(a_{p} \chi_{p}^{\prime}+a_{w} \chi_{w}^{\prime}\right)\right. \\
& \left.+\mathrm{i}\left[\left(1+a_{p}\right) \dot{\chi}_{p}^{\prime}+\left(1+a_{w}\right) \dot{\chi}_{w}^{\prime}\right]\right\} .
\end{aligned}
$$

There are two complex integration constants to be chosen, defining the amplitudes and phases of the two free terms Chandler and FCN. Since Chandlerian motion in celestial frame is quasi-diurnal, it belongs to a 'forbidden' frequency and as such should be suppressed. Therefore, we put $a_{\mathrm{C}}$ of Eqs. (3) equal to zero, which leads to the condition $\dot{P}(0)=$ $\mathrm{i} \sigma_{f}^{\prime} P(0)$ and, consequently, to $Y_{1}(0)=P(0), Y_{2}(0)=\mathrm{i}\left(\sigma_{f}^{\prime}-\right.$ $\left.\sigma_{\mathrm{C}}^{\prime}\right) P(0)$. To integrate Eqs. (4), we used fourth-order RungeKutta method; subroutine rk4 (Press et al. 1992) re-written into a complex form is applied.

2. MHB transfer function, expressing the frequency-dependent ratio between non-rigid and rigid Earth nutation terms (Mathews et al. 2002) reads

$T_{\mathrm{MHB}}(\sigma)=\frac{e_{R}-\sigma}{e_{R}+1} N_{\circ}\left[1+(1+\sigma)\left(Q_{\circ}+\sum_{j=1}^{4} \frac{Q_{j}}{\sigma-s_{j}}\right)\right]$,

where $\sigma$ is the frequency of nutation, $e_{R}$ is the dynamical ellipticity of the rigid Earth, $N, Q$ are complex constants, and $s_{j}$ are complex resonance frequencies, corresponding to:

1. Chandler wobble $-\mathrm{CW}\left(P_{\text {ter. }} \approx 435 \mathrm{~d}\right)$;

2. retrograde free core nutation $-\operatorname{RCFN}\left(P_{\text {cel. }} \approx 430 \mathrm{~d}\right)$;

3. prograde free core nutation $-\operatorname{PFCN}\left(P_{\text {cel. }} \approx 1020 \mathrm{~d}\right)$;

4. inner core wobble $-\mathrm{ICW}\left(P_{\text {ter. }} \approx 2400 \mathrm{~d}\right)$.

Numerical values of the constants of MHB transfer function are given in Table 1.

Unlike the broadband Liouville equations above, all frequencies here are given in terrestrial frame in cycles per sidereal day [cpsd]. It is clear that namely the nutation terms with frequencies close to resonances $s_{j}$ are affected. As $s_{1}$ is far from the nutation frequencies and resonances due to $s_{3}, s_{4}$ are very weak, a dominant role plays the RFCN resonance whose frequency $s_{2}$ is related to $\sigma_{f}^{\prime}$ simply as $s_{2}=\sigma_{f}^{\prime} / \Omega-1$. Thus, 
Table 1. Constants of MHB transfer function.

\begin{tabular}{rrr}
\hline \hline Parameter & $\mathrm{Re}$ & $\mathrm{Im}$ \\
\hline$e_{R}$ & 0.0032845075 & - \\
$N_{\circ}$ & 1.00001224 & - \\
$Q_{\circ}$ & -0.165291 & 0.0318995 \\
$Q_{1}$ & -0.948081 & 0.0678857 \\
$s_{1}$ & $3.11279 \times 10^{-3}$ & $3.76098 \times 10^{-4}$ \\
$Q_{2}$ & $4.89324 \times 10^{-2}$ & $1.61700 \times 10^{-3}$ \\
$s_{2}$ & -1.00231811 & $2.50607 \times 10^{-5}$ \\
$Q_{3}$ & $2.96114 \times 10^{-4}$ & $-9.56740 \times 10^{-5}$ \\
$s_{3}$ & -0.999026445 & $7.78663 \times 10^{-4}$ \\
$Q_{4}$ & $-1.10856 \times 10^{-5}$ & $-1.22654 \times 10^{-6}$ \\
$s_{4}$ & $4.1324 \times 10^{-4}$ & $9.28220 \times 10^{-8}$ \\
\hline
\end{tabular}

the closer is the frequency of a nutation term to $s_{2}$, the larger is the relative change of its amplitude. Since $T_{\mathrm{MHB}}$ is a complex function, not only the absolute values of the amplitudes, but also their phases are changed. To illustrate the change of nutation amplitudes, we show in Fig. 1 the real part of $T_{\mathrm{MHB}}$ as function of terrestrial frequency $\sigma$; the frequencies of five nutation terms that are most affected are also depicted. The frequencies in terrestrial frame are all retrograde quasidiurnal. In celestial frame, the frequencies are long-periodic, to the left of $-1 \mathrm{cpsd}$ retrograde, to the right prograde. The values of transfer function differ from unity significantly, and especially the annual retrograde nutation term is affected. It is clear that if $s_{2}$ is changed, the value of $T_{\mathrm{MHB}}$ for the same frequency $\sigma$ and the amplitude of corresponding nutation term also change. We used specifically this resonant effect to re-calculate nutation and, consequently, also the CPO for each combination $T_{f}, Q_{f}$. These corrected CPO values are then used to compute the least-squares fit to integrated pole positions. To this end, we used only the five afore mentioned nutation terms that are most affected by the change of $s_{2}$; the effect of the remaining ones is negligible.

\section{Input data used and their modifications}

In this study, we used data covering the 30-yr interval 1986.02016.0. They are described below, together with all necessary modifications.

\subsection{Celestial pole offsets}

Celestial pole offsets $\mathrm{d} X, \mathrm{~d} Y$, referred to IAU 2000 nutation model, were taken from IERS C04 combined solution (available at IERS Earth Orientation Center). They are given in one-day steps. The data were filtered to contain only periods between 10 and 6000 days, using the filter by Vondrák (1977). Shorter periods are supposed to be noise, longer ones caused by other than geophysical fluids. They were further treated as complex values $\mathrm{d} X+\mathrm{id} Y$. As already noted in the preceding Section, Sun-synchronous correction, equal to $(0.1082+0.0104 \mathrm{i}) \mathrm{e}^{\mathrm{i} l^{\prime}}$ mas, where $l^{\prime}$ is the mean anomaly of the Sun, was removed. Further corrections due to $\mathrm{FCN}$ resonance were applied for each individual combination of $T_{f}, Q_{f}$. This corrected CPO series is then used as a standard with which the integrated values are compared.

\subsection{Geophysical excitations}

Equatorial components of excitations by geophysical fluids $\chi_{1}$, $\chi_{2}$ for two different models are represented by the effective angular momentum functions (EAMF) in six-hour steps. We use both matter and motion terms, available from IERS Global Geophysical Fluids Center. They were re-calculated from terrestrial to celestial frame, smoothed to contain only periods longer than ten days, and centered. Time derivatives, necessary to integrate Eq. (1), were also calculated. The series that we use in this study are as follows:

- atmospheric EAMF with inverted barometer correction (IB, representing a simple oceanic model), based on the US meteorological centers data NCEP/NCAR (National Center for Enviromental Prediction/National Center for Atmospheric Research, Zhou et al. 2006);

- atmospheric EAMF based on the ECMWF (The European Centre for Medium-Range Whether Forecast); atmospheric excitations ERA40 (1986.0-2013.0) and ERA operational (2013.0-2016.00) and oceanic excitation, based on the Ocean Model for Circulation and Tides (OMCT), driven by the aforementioned atmosphere (Thomas 2002). These EAMFs are prepared in GFZ Potsdam (Dobslaw et al. 2010).

It was recently noticed that sudden jumps of phase and/or amplitude of Earth orientation are somehow correlated with GMJ, which are rapid changes of the second time derivative of geomagnetic field (see, e.g., Mandea et al. 2010). Specifically, the correlation in case of FCN was noticed by Malkin (2013), and later confirmed by Vondrák \& Ron (2015). Since the exact mechanism of transferring the changes of geomagnetic field to Earth's orientation remains unknown, we were unable to compute the excitation of GMJ directly from the known changes of geomagnetic field. Instead, we modelled it by impulse-like function, centered at GMJ epochs, whose amplitude is estimated to yield the best fit between the excited and observed nutation. To model the excitation by GMJ in celestial frame, we used an additional excitation of the following form, applied in the interval $t_{\circ} \pm \Delta / 2$

$\chi_{\mathrm{GMJ}}^{\prime}=\frac{a}{2}\left[1+\cos \frac{2 \pi\left(t-t_{\circ}\right)}{\Delta}\right]$,

in which we made $t_{\circ}$ equal to GMJ epochs and the width $\Delta=$ 200 days, the values that we found to be appropriate in our preceding studies.

It should be stressed that the specific shape of the excitation (6) chosen here is not substantial for the final result; we used stepwise and triangular shapes in the past, with similar result. We use this very form only because we felt that the excitation should be smooth and continuous, with the length comparable to the duration of GMJ. This additional excitation corresponds to a retrograde diurnal signal in terrestrial frame, with the amplitude smoothly increasing to $a$ and then decreasing during 200 days.

Seven epochs of geomagnetic jerks are used: 1991.0, 1994.0, 1999.0, 2007.5 (given by Malkin 2013), 2003.5, 2004.7 (after Olsen \& Mandea 2008; Mandea et al. 2010), and 2011.0 (Chulliat \& Maus 2014). Similarly to our recent work on Chandler wobble parameters (Vondrák et al. 2017), we use a procedure consisting of several steps. First, the initial pole position $P(0)$ is found to yield the best fit to observations in the interval between the beginning (1986.0) and the first GMJ epoch. Then, the complex amplitude $a$ at the first GMJ epoch is chosen so that it gives the best fit in the interval between the first and second GMJ epochs. The same procedure is then repeated for all GMJ epochs until the end of the whole interval (2016.0) is reached. 
Table 2. Complex amplitudes of GMJ excitations [mas].

\begin{tabular}{rrrrrrr}
\hline \hline Epoch & \multicolumn{2}{c}{ NCEP } & \multicolumn{2}{c}{ ERA/OMCT } & \multicolumn{2}{c}{ SSC } \\
\hline 1991.0 & -0.92 & 0.15 & -0.48 & -0.35 & -0.88 & -0.49 \\
1994.0 & -0.15 & 0.48 & 0.53 & 2.22 & 0.07 & -0.01 \\
1999.0 & -0.57 & 0.59 & 0.39 & -0.38 & -1.57 & 0.14 \\
2003.5 & 0.44 & -1.05 & 0.92 & -2.13 & 0.35 & -0.76 \\
2004.7 & 0.54 & -0.67 & 1.19 & -0.06 & 0.59 & -0.50 \\
2007.5 & -1.04 & -1.14 & -2.18 & -0.87 & -0.88 & -1.05 \\
2011.0 & -0.94 & -0.82 & -2.88 & -0.96 & -0.89 & -0.75 \\
\hline
\end{tabular}

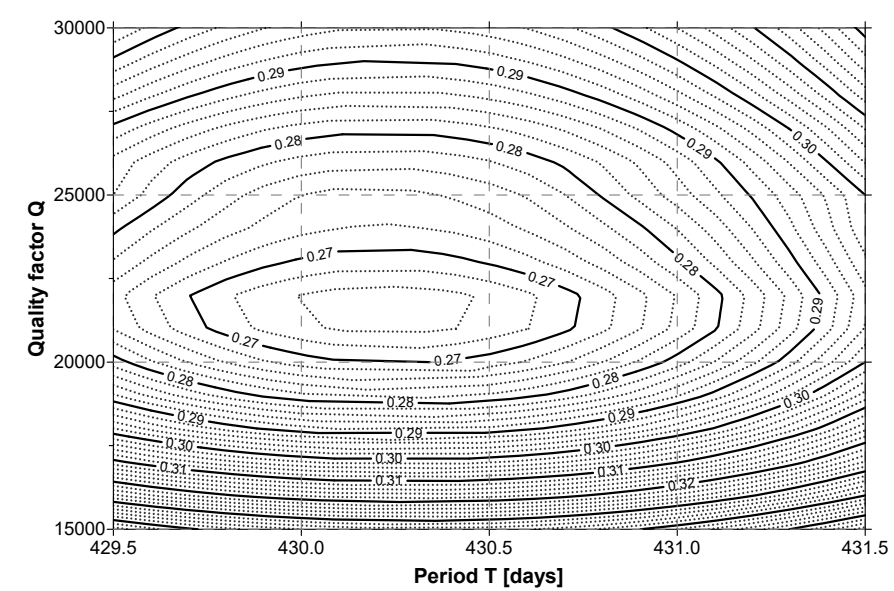

Fig. 2. Rms fit between integrated and observed celestial pole offsets; NCEP IB excitations are used.

\section{Results}

Here we show the integrated results for both series of atmospheric and oceanic excitations mentioned above (NCEP IB and ERA/OMCT, respectively), and also for only Sun-synchronous correction, as a special simplified model of geophysical excitations. In each of these solutions, we show the results with and without GMJ effects. The amplitudes of GMJ excitations, obtained by the method described at the end of Sect. 3, are displayed in Table 2. Their magnitudes are comparable to those caused by the atmosphere.

To derive final values of $T_{f}, Q_{f}$ and their uncertainties, we used traditional least-squares approximation, applied near the minima estimated from the plots $\left(T_{f}, Q_{f}\right)$ in Figs. 2, 3, 5, 6, 8, and 9. Partial derivatives of the individual integrated $\mathrm{CPO}$ values with respect to unknowns $T_{f}, Q_{f}$, needed to form observation equations, are computed numerically. To this end, we repeated the integration four times, with slightly different values $T_{f}, Q_{f}$. Right-hand sides of observational equations are the differences between integrated and observed CPO values. Normal equations were then formed and standard least-squares procedure was applied to find the final values and formal uncertainties of the unknowns. This procedure, however, seems to yield formal uncertainties that are probably too optimistic, when compared with the values obtained by other authors.

\subsection{NCEP/NCAR}

The rms fit of the numerical integration with NCEP IB excitations to CPO is depicted as a contour plot in Fig. 2. The minimum rms is achieved for $T_{f}=430.23 \pm 0.05 \mathrm{~d}, Q_{f}=$ $21800 \pm 300$, with rms $=0.267$ mas. Correlation between the two series is 0.440 .

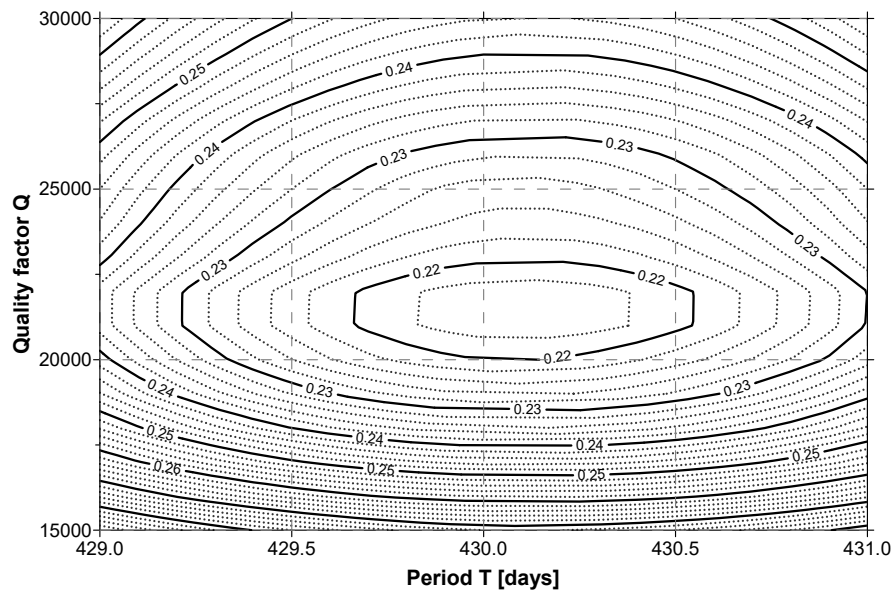

Fig. 3. Rms fit between integrated and observed celestial pole offsets; NCEP IB excitations plus GMJ effects are used.

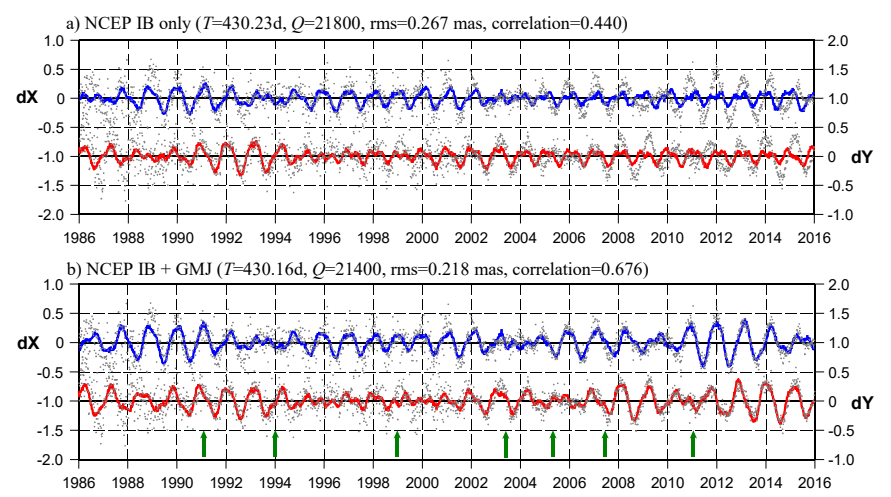

Fig. 4. Integrated (full line) and observed (gray dots) celestial pole offsets for the best-fitting FCN parameters. NCEP IB excitations (upper plot) and NCEP IB + GMJ excitations (lower plot) are used.

The situation is only slightly different if GMJ effects are added, as shown in Fig. 3. The best rms fit is obtained for $T_{f}=430.16 \pm 0.04 \mathrm{~d}, Q_{f}=21400 \pm 200$, with rms $=0.218$ mas and correlation 0.676 . When compared with the preceding case, the parameters $T_{f}, Q_{f}$ are a little different, their uncertainties are smaller and both the fit and correlations are much improved.

The integrations for the best-fitting parameters mentioned above are compared with the observed CPO in Fig. 4. GMJ epochs in the lower plot are indicated. The improvement of the fit between the two series, brought about by additional GMJ excitations is evident.

\subsection{ERA/OMCT}

A contour plot of the rms fit between the integrated and observed CPO with ERA/OMCT atmospheric plus oceanic excitations is shown in Fig. 5. The best fit is obtained for $T_{f}=430.23 \pm 0.08 \mathrm{~d}$, $Q_{f}=18700 \pm 300, \mathrm{rms}=0.422$ mas and the correlation between the two series is very weak, only 0.028 . This solution is evidently much worse than the one with NCEP IB excitations.

The situation is however much improved if additional excitations due to GMJ are applied. It is demonstrated in Fig. 6. The best values of FCN parameters are $T_{f}=429.96 \pm 0.05 \mathrm{~d}$, $Q_{f}=19800 \pm 200, \mathrm{rms}=0.242$, and correlation grew to 0.617 .

The integrations for the best-fitting parameters and ERA/OMCT atmospheric and oceanic excitations are compared with the observed CPO in Fig. 7; again, GMJ epochs in the lower 


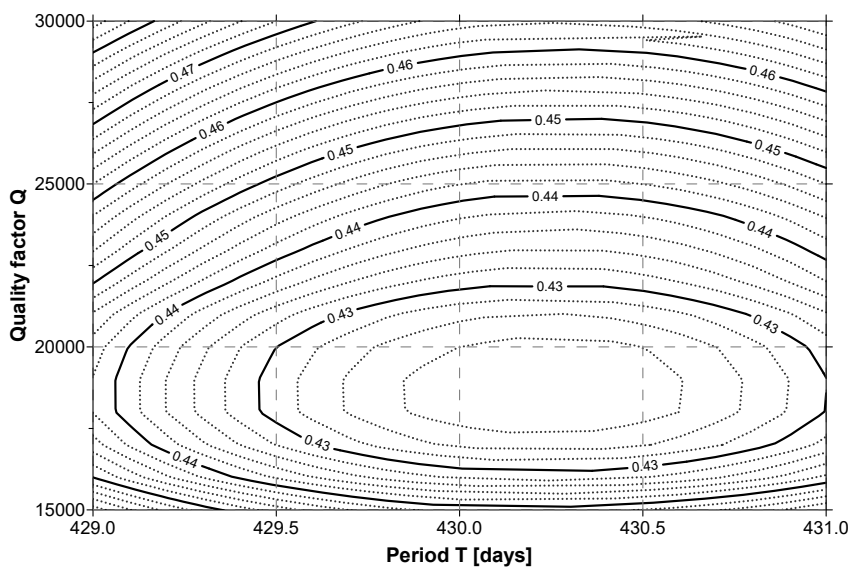

Fig. 5. Rms fit between integrated and observed celestial pole offsets; ERA/OMCT excitations are used.

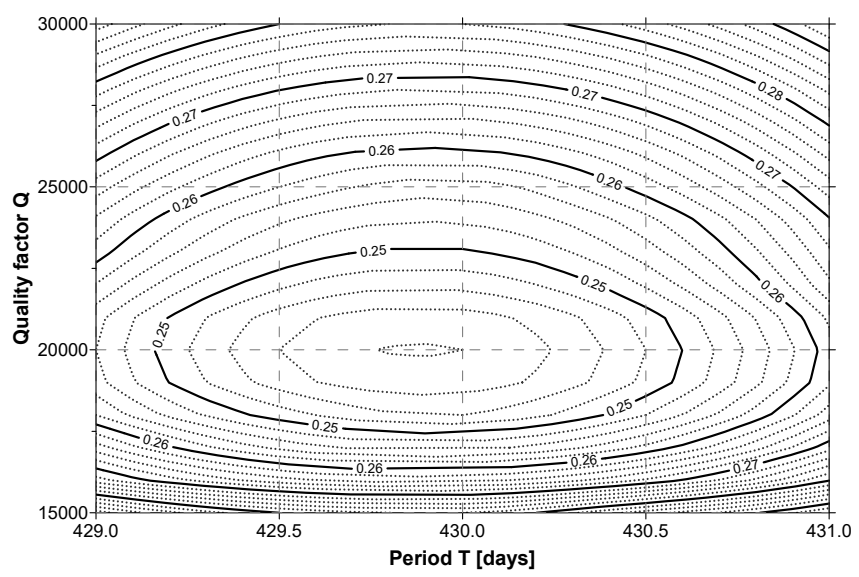

Fig. 6. Rms fit between integrated and observed celestial pole offsets; ERA/OMCT excitations plus GMJ effects are used.

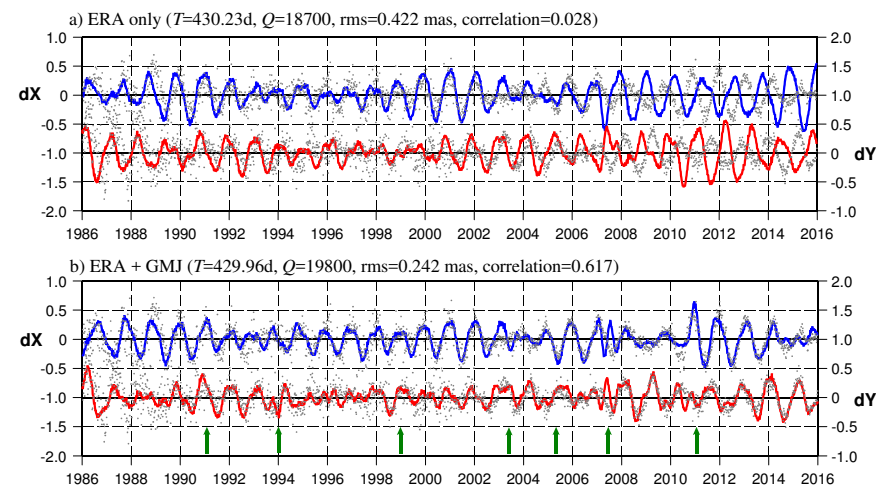

Fig. 7. Integrated (full line) and observed (gray dots) celestial pole offsets for the best-fitting FCN parameters. ERA/OMCT atmospheric and oceanic excitations (upper plot) and ERA/OMCT + GMJ excitations (lower plot) are used.

plot are indicated. The improvement of the fit between the two series, caused by additional GMJ excitations, is significant.

\subsection{Sun-synchronous correction}

This is a specific case (instead of real geophysical excitations a simple empirical term is used), however we treat it similarly to preceding atmospheric and oceanic excitations. We put these

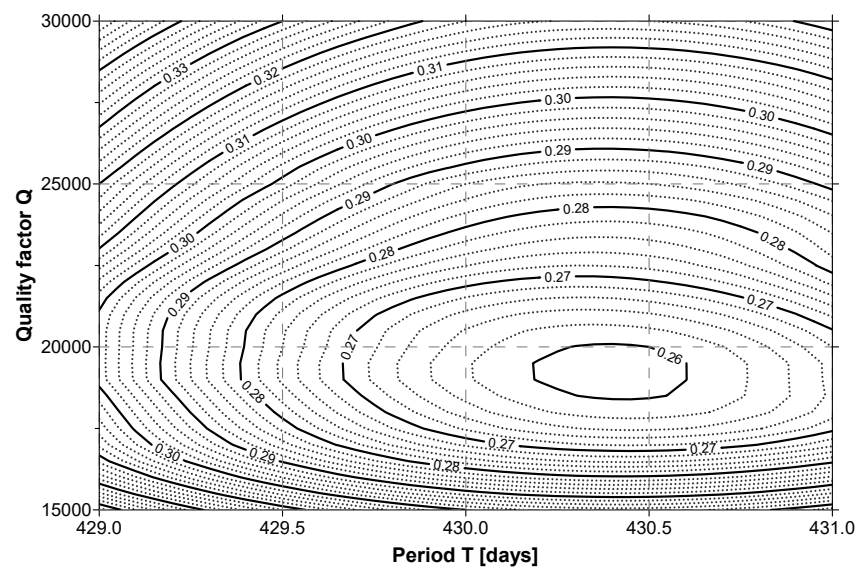

Fig. 8. Rms fit between integrated and observed celestial pole offsets; Sun-synchronous correction is used.

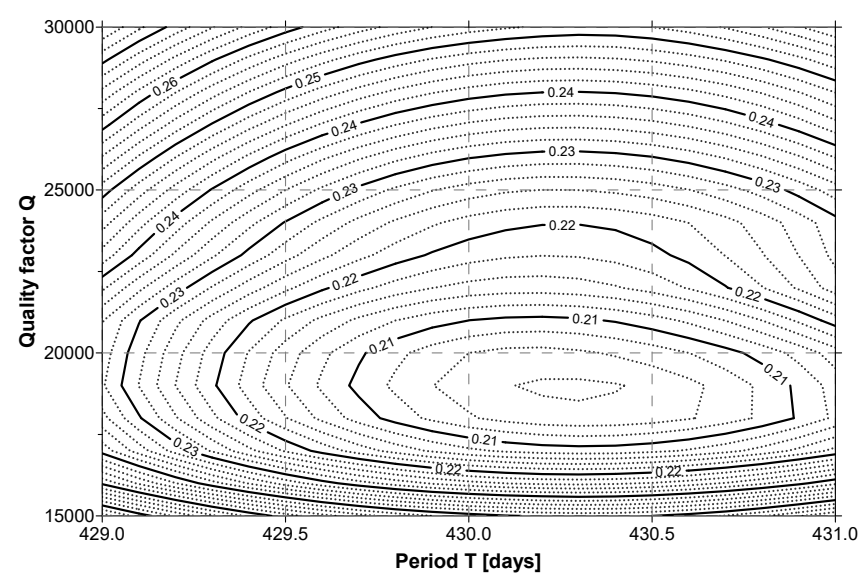

Fig. 9. Rms fit between integrated and observed celestial pole offsets; Sun-synchronous correction plus GMJ effects are used.

excitations equal to zero, so we integrate in fact only the free part and, at the end, we add back the Sun-synchronous correction (SSC) to the result. The resulting contour plots, representing rms fits both without and with GMJ effects, are shown in Figs. 8 and 9 , respectively. The fits are surprisingly very good when compared with NCEP and ERA/OMCT excitations. FCN parameters are $T_{f}=430.37 \pm 0.05 \mathrm{~d}, Q_{f}=19300 \pm 200, \mathrm{rms}=0.259$ mas and correlation 0.465 for SSC alone, and $T_{f}=430.28 \pm 0.04 \mathrm{~d}$, $Q_{f}=19500 \pm 200, \mathrm{rms}=0.204$ mas and correlation 0.716 if GMJ excitations are added. Not unlike in the preceding two cases, the fit is significantly improved when the GMJ effects are added.

Again, we compare the result of the integration with the observed CPO in Fig. 10. The significant improvement caused by including additional GMJ excitations is evident from comparison of upper and lower plot.

\section{Discussion and conclusions}

Our newly proposed method of determining parameters of FCN proves to be a good tool to determine both period and $Q$-factor of the free term on nutation. It is clear that geophysical excitations yield small but now measurable contribution to nutation. Overview of all results obtained by this method in the preceding section is given in Table 3. It seems that the formal uncertainties 
Table 3. Results of FCN period $T_{f}$ (in days) and quality factor $Q_{f}$, obtained with only atmospheric and oceanic excitations (A+O), and GMJ effects added $(\mathrm{A}+\mathrm{O}+\mathrm{G})$.

\begin{tabular}{l|cccc|cccc}
\hline \hline & \multicolumn{4}{|c|}{$\mathrm{A}+\mathrm{O}$} & \multicolumn{4}{c}{$\mathrm{A}+\mathrm{O}+\mathrm{G}$} \\
Excitations & $T_{f}$ & $Q_{f}$ & $\mathrm{rms}$ & corr. & $T_{f}$ & $Q_{f}$ & rms & corr. \\
\hline NCEP IB & $430.23 \pm 0.05$ & $21800 \pm 300$ & 0.267 & 0.440 & $430.16 \pm 0.04$ & $21400 \pm 200$ & 0.218 & 0.676 \\
ERA/OMCT & $430.23 \pm 0.08$ & $18700 \pm 300$ & 0.422 & 0.028 & $429.96 \pm 0.05$ & $19800 \pm 200$ & 0.242 & 0.617 \\
SSC & $430.37 \pm 0.05$ & $19300 \pm 200$ & 0.259 & 0.465 & $430.28 \pm 0.04$ & $19500 \pm 200$ & 0.204 & 0.716 \\
\hline
\end{tabular}

Notes. Rms fits (in mas) between integrated and observed CPO values and correlations between both series are also shown.

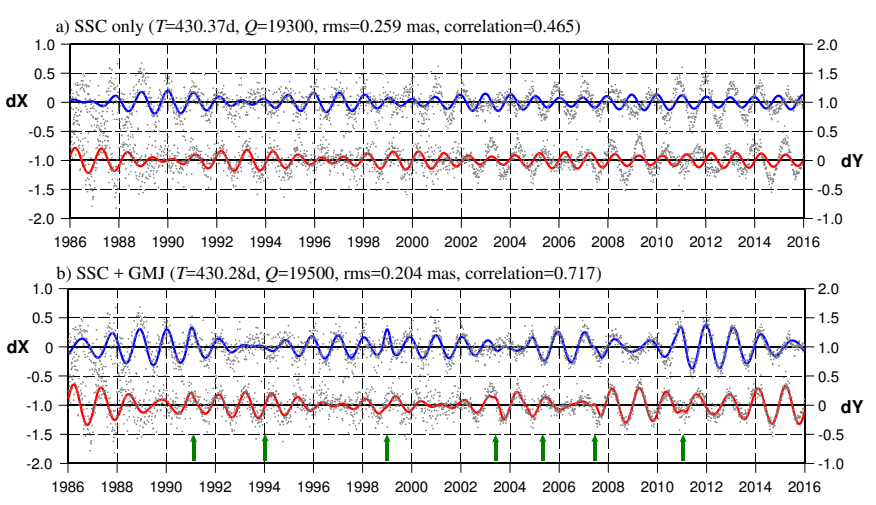

Fig. 10. Integrated (full line) and observed (gray dots) celestial pole offsets for the best-fitting FCN parameters. Empirical SSC plus best fitting free term (upper plot) and SSC + GMJ excitations (lower plot) are used.

that we obtained from the least-squares solution are too optimistic; their real values are probably somewhat higher. The values for our error bars are much smaller than those in the literature cited in Introduction. This can be ascribed partly to the fact that our new results are based on newer and more accurate values of both celestial pole offsets and geophysical excitations, partly to much longer time span used (30 yr in our case), and also due to the new combined method used in this study. For example, compared with our older results (Vondrák et al. 2005) based on the traditional resonance approach and data covering only $20 \mathrm{yr}$ $\left(T_{f}=430.55 \pm 0.11 \mathrm{~d}, Q_{f}=19900 \pm 500\right)$, our new results have error bars more than two times smaller, even if only traditional atmospheric and oceanic excitations are considered. The inclusion of additional (somewhat hypothetical) GMJ effects brings about an improvement of only some 20 per cent, so the improvement is only marginally caused by this effect.

Generally speaking, an improvement is achieved in all three solutions when GMJ effect is taken into account - all uncertainties become smaller and correlations higher (compare the left and right part of the table). It is also clear from the table that inclusion of GMJ effects has a tendency of yielding slightly lower values of the period $T_{f}$. ERA/OMCT atmospheric and oceanic contribution seems to provide too high amplitudes which is reflected in a worse fit between integrated and observed celestial pole offsets. This is clearly seen in Fig. 7 (when compared with Figs. 4 and 10), and also from the uncertainties and correlations presented in Table 3 . Better results are obtained with NCEP IB excitations. We expected this, since our preceding studies (Vondrák \& Ron 2014) already indicated the preference of NCEP excitations, namely for the nutation.

However, quite unexpectedly, the solution with MHB Sunsynchronous correction yields even better results. This might probably reflect the fact that atmospheric/oceanic excitations are still not completely reliable and have large uncertainties. The simple SSC model, based on VLBI observations before 2000, describes the excitations by all geophysical fluids (i.e., atmosphere + oceans + hydrosphere) better than those based on atmospheric + oceanic modeling. In addition, the simple SSC model seems to be still valid, even for epochs much later than 2000 .

The FCN parameters, obtained from all our solutions and shown in Table 3, are not much different, individual values seem to be mutually consistent. Nevertheless, our preferred values are those with the best fit and obtained with highest accuracy, that is, based on SSC + GMJ excitations: $T_{f}=430.28 \pm 0.04 \mathrm{~d}$ and $Q_{f}=19500 \pm 200$.

Acknowledgements. This study was carried out thanks to the grant No. 1315943S, awarded by the Grant Agency of the Czech Republic, and also supported by the project RVO:67985815.

\section{References}

Amoruso, A., Botta, V., \& Crescentini, L. 2012, Geophys. J., 189, 923 Barnes, R. T. H., Hide, R., White, A. A., \& Wilson C. A. 1983, Proc. R. Soc. Lond. A, 9, 387, 31

Breziński, A. 1994, Manuscripta geodaetica, 19, 157

Capitaine, N., \& Wallace, P. T. 2006, A\&A, 450, 855

Chao, B. F., \& Hsieh, Y. 2015, Earth Planet. Sci. Lett., 432, 483

Chulliat, A., \& Maus, S. 2014, J. Geophys. Res. Solid Earth, 119, 1531

Defraigne, P., Dehant, V., \& Hinderer, J. 1999, J. Geophys. Res., 99, 9203

Dobslaw, H., Dill, R., Grotzsch, A., Brzezinski, A., \& Thomas, M. 2010, J. Geophys. Res., 115, B10406

Florsch, N., \& Hinderer, J. 2000, Phys. Earth Planet. Interior, 117, 21

Gubanov, V. S. 2010, Astron. Lett., 36, 444

Hinderer, J., Boy, J. P., Gegout P., et al. 2000, Phys. Earth Planet. Interior, 117, 37

Huang, C. L., Dehant, V., Liao, X. H., Van Holst, T., \& Rochester, M. G. 2011, J. Geophys. Res., 116, B03403

Koot, L., \& de Viron, O. 2011, Geophys. J. Int., 185, 1255

Krásná, H., Böhm, J., \& Schuh, H. 2013, A\&A, 555, A29

Malkin, Z. 2013, J. Geodyn., 72, 53

Mandea, M., Holme, R., Pais, A. et al. 2010, Space Sci. Rev., 155, 147

Mathews, P. M., Herring, T. A., \& Buffet, B. A. 2002, J. Geophys. Res., 107, B4

Olsen, N., \& Mandea, M. 2008, Nature Geosci., 1, 390

Petit, G., \& Luzum, B. 2010. IERS Conventions 2010, IERS Tech. Note 36 (Frankfurt a. M., Germany: Ver. Bundesamts für Kartographie und Geodaesie)

Press, W. H., Teukolsky, S. A., Vetterling, W. T., \& Flannery, B. P. 1992, Numerical Recipes in Fortran 77, The Art of Scientific Computing, 2nd Edn (Cambridge University Press)

Rosat, S., \& Lambert, S. B. 2009, A\&A, 503, 287

Souchay, J., Loysel, B., Kinoshita, H., \& M. Folgueira 1999, A\&AS, 135, 111

Thomas, M. 2002, Ph.D. dissertation, University of Hamburg, Germany

Vondrák, J. 1977, Bull. Astron. Inst. Czechosl., 28, 84

Vondrák, J., \& Ron, C. 2014, Acta Geodyn. Geomater., 11, 193

Vondrák, J., \& Ron, C. 2015, Serb. Astron. J., 191, 59,

Vondrák, J., Weber, R., \& Ron, C. 2005, A\&A, 444, 297

Vondrák, J., Ron C., \& Chapanov, Ya. 2017, Adv. Space Res., 59, 1395

Zhou, Y. H., Salstein, D. A., \& Chen, J. L. 2006, J. Geophys. Res., 111, D12108,

Zhou, Y. H., Qiang, Z., Salstein, D., et al. 2016, Adv. Space Res., 57, 2136 